\title{
Challenges in the Performance of Awake Craniotomies in the Philippine General Hospital: A Case Series
}

\author{
Geraldine Raphaela B. Jose, MD¹ and Gerardo D. Legaspi, MD² \\ ${ }^{1}$ Department of Anesthesiology, Philippine General Hospital, University of the Philippines Manila \\ ${ }^{2}$ Division of Neurosurgery, Department of Neurosciences, Philippine General Hospital, University of the Philippines Manila
}

\begin{abstract}
Awake craniotomy is a neurosurgical technique that involves an awake neurological testing during the resection of an intracranial lesion in eloquent cerebral cortical areas representing motor, language, and speech. This technique is highlighted by an intra-operative cortical mapping that requires active participation by the patient and poses unique challenges to the anesthesiologist. The surgical and anesthetic techniques have evolved significantly over time, as the neurosurgeon and the anesthesiologist learn new steps in making this technique safe to achieve reasonable patient satisfaction. A thorough understanding of this surgical technique's rationale will guide the anesthesiologist in planning the anesthetic management depending on the surgery and neurologic testing. Constant communication between the neurosurgeon, anesthesiologist, and the patient will define this surgical technique's success. It is already a well-established procedure; however, factors that contribute to failures in awake craniotomy procedures have not been well characterized in the literature. Failure is defined as the inability to conduct awake neurologic testing during the awake craniotomy procedure because of various factors which will be described. This paper aims to review the challenges in the performance of three (3) cases of awake craniotomies performed in the Philippine General Hospital. The challenges described in these three (3) cases reveal that this can be experienced by the neurosurgeon, neuroanesthesiologist, and most especially the patient in an acute critical condition. Identification of the procedures' failure and the steps taken to manage such situations with the patient's safety in mind are discussed.
\end{abstract}

Keywords: awake craniotomy, awake neurologic testing, awake craniotomy failures, challenges in awake craniotomy, monitored anesthesia care, total intravenous anesthesia, target-controlled infusion

\section{INTRODUCTION}

Awake craniotomy is a surgical technique for resectioning intracranial mass lesions located in eloquent areas of the brain. The eloquent areas are the motor, language, and speech cortices, including adjacent areas responsible for other functions such as frontal lobe-executive activity. It is an intracranial surgical procedure wherein the patient is purposely kept awake at certain phases of the surgery, particularly during cortical mapping and resection of the intracranial lesion. The reason for this is to minimize the functional neurologic consequences of cerebral tissue resection by having the patient participate in an awake neurologic testing.

\section{HISTORY OF AWAKE CRANIOTOMY IN THE PHILIPPINE GENERAL HOSPITAL}

Corresponding author: Geraldine Raphaela B. Jose, MD Department of Anesthesiology Philippine General Hospital

University of the Philippines Manila

Taft Avenue, Manila 1000 Philippines

Email: gbjose@up.edu.ph
In the 1950s, the awake craniotomy technique was introduced in the Philippines by Dr. Victor Reyes, a neurosurgeon in the Philippine General Hospital (PGH). He utilized very "crude" neurologic testing, and his outcomes have not been reported. Since then, there were no awake 
craniotomy procedures performed in the PGH. In 2006, Dr. Gerardo Legaspi, a PGH neurosurgeon, performed an awake craniotomy and excision of a low-grade astrocytoma at the left frontal lobe without any cortical stimulator. Brain mapping was done using a low-frequency monopolar stimulation on the cortical surface (a PGH adaptation) and asking the patient to move extremities during the resection. From 2006 to 2015, awake surgery was done without cortical stimulation. Guidance during surgical resection was a functional performance with gentle brain manipulation. A 1 to $2 \mathrm{~cm}$ peri-lesional margin was maintained during the excision. Fortunately, surgical outcomes were good and were supported by post-op brain MRI revealing complete excision of the lesion with a right postoperative course. In 2015, Dr. Mitchell Berger, a neurosurgeon from the University of California in San Francisco (UCSF), introduced various cortical mapping techniques for an awake craniotomy procedure to the neurosurgeons of the PGH. Yearly after that, he came to the Philippines to share his expertise in brain mapping and white mater anatomy, which the Filipino neurosurgeons learned to appreciate. Cortical mapping was then adapted in the performance of the awake craniotomy procedure. There were two (2) schools of cortical mapping techniques introduced. Firstly, performing a large craniotomy flap and doing systematic cortical mapping until a positive response is elicited using a cortical stimulator; secondly, by doing a limited craniotomy and use a high-frequency cortical stimulation until no neurologic response is achieved. A positive response is an involuntary movement or a change in speech when the cortical surface is stimulated. In 2017, cortical ultrasound was introduced in the awake craniotomy technique to better understand the cortical lesion's depth and the extent of the surgical excision. ${ }^{1}$

From 2006 to 2019, there were 72 cases of awake craniotomy procedure in the PGH. The age range was 16 to 64 years of age. The incidence by the pathology of the awake craniotomy procedures in the PGH are as follows: Glioblastoma Multiforme - GBM (34\%); Low Grade/ Diffuse Astrocytoma (18\%); Metastatic Adenocarcinoma (14\%); Oligodendroglioma (8\%); Meningioma (8\%); Arteriovenous - AV Malformation (8\%); Cavernous Malformation (6\%); Anaplastic Astrocytoma (2\%) and Cerebral Aneurysm (2\%). ${ }^{1}$

The surgical methods to enhance the extent of resection and minimize neurologic morbidity have evolved as a more in-depth understanding of the procedure and its consequences have been acquired. In the beginning, intraoperative surgical planning and assessment were based on microscopy, neuro-navigation, and cortical mapping. Recently, intra-operative MRI/CT, brain ultrasound, fluorescence guidance, and endoscopy play vital roles in the surgical outcome. The introduction of new anesthetic agents with a more dependable onset and duration of action has enormously improved the anesthetic technique. An in-depth understanding of the "surgical landscape of the procedure," as well as the anesthetics' pharmacology, has served as a guide for the neuroanesthesiologist in timing the anesthetics at certain phases of the procedure, which are very critical in the success of the surgery. Brain monitoring devices that determine anesthesia's depth serve as a useful guide in awakening the patient at appropriate times. To perform an awake craniotomy procedure, it is necessary to select patients and the surgical and anesthesia team's skills. The lack of cooperation and sedation failure will significantly shift to a general anesthesia inhalation anesthesia technique.

The main goal in awake craniotomy is to maximize intracranial lesion resection while keeping neurological functions as intact as possible by awake neurologic testing during the operation. Sensori-motor mapping of the involved cortex is performed using a cortical stimulator. This is used to mark the cortical areas which are clinically silent and where surgical resection can be achieved with minimal risk. Better surgical outcomes are observed in awake craniotomy, which leads to a shorter hospital stay and better survival.In comparing awake craniotomy from the conventional craniotomy with tumor excision, the neurologic deficits are (7\% vs. $23 \%)$ and hospital stay is (1.7 vs. 9 days), respectively. This data suggests that awake craniotomy could improve access to neurosurgical care in poorer countries. ${ }^{2}$ Also, after an awake craniotomy, the patient has less pain, less narcotic usage, and reduced postoperative nausea and vomiting. Although this procedure can be stressful, with up to $30 \%$ of the patients having considerable pain and $10 \%$ to $14 \%$ experiencing significant anxiety, patient acceptance, and postoperative satisfaction are high. ${ }^{3}$ Post-traumatic stress disorder does not seem to occur. ${ }^{4}$

Awake craniotomy for tumor resection presents many challenges for the neurosurgeon and the anesthesiologist, and the patient. Adequate analgesia and sedation must be provided at certain phases of the procedure while allowing them to be conscious and cooperative during neurological testing. ${ }^{5}$ The success of an awake craniotomy procedure will depend mainly on an in-depth knowledge of the procedure's surgical phases and its concomitant anesthetic requirements. Several analgesias, sedation, and anesthetic techniques have been described, differing in the drugs administered, how they are delivered, and the airway management. ${ }^{6}$ These techniques include asleep-awake-asleep (SAS) and asleep-awake-awake (SAA) techniques.

Awake craniotomy requires skill, experience, and the commitment of the entire surgical and anesthesia team. A good surgical outcome depends mainly on thorough preoperative preparation. A proper psychological evaluation of the patient is warranted to be ready for an awake surgical technique's demands. Not surprisingly, most patients are anxious about being awake during craniotomy and may have significant misconceptions about the procedure. A frank description of what they can expect to hear, see, and feel during the procedure usually eases anxiety. Preoperative assessment of language function and vision needs to be done to assess his abilities to perform mapping related tasks. In addition to routine preoperative evaluation, the patient assessment 
includes evaluating the ability to tolerate the procedure, cognitive function, seizure morphology, and incidence of postictal behavioral problems or confusion. Patients who cannot cooperate and those with significant dysphasia may not be candidates for awake craniotomy. Careful patient selection and extensive preoperative preparation are thought to be critical in this population. Preoperative patient interview by all team members is essential in building trust and engagement. Establishing good rapport among the team members provides the patient with the reassurance and empathy that the patient needs. The patient must be informed of the duration of the surgery, the indications for waking up during the excision segment of the procedure, and also the actual intra-operative scenario, which include: what to expect as he enters the operating room, positioning during craniotomy, application of headpins, and craniotomy noise. Another critical discussion that has to be made with the patient is the possible events that may happen during the actual surgery and the likelihood of conversion to general anesthesia as deemed fit.

The anesthetic regimen for an awake craniotomy is designed to anticipate the degree of surgical stimulation with the patient's response and safety in every phase of the surgical procedure. There are several phases of the procedure which, may be described as the "surgical landscape of an awake craniotomy." This would dictate the level of anesthesia required in every phase. This comprises the following: (1) head pinning; (2) skin incision; (3) bone flap elevation; (4) dural opening; (5) excision of the intracranial lesion (awake phase); (6) dural closure; (7) return of bone flap; and (8) skin closure. The most stimulating phases are phases 1 to 4 . In phase 5 , we expect the patient to be awake for neurologic testing. There are occasions of Visual Analog Scale (VAS) 4-5 of non-specific headache brought about by meningeal irritation which can be relieved by giving short-acting opioid IV. In phases 6 to 8 , the patient may be maintained on moderate sedation to achieve a pain-free craniotomy closure. Challenges in the performance of an awake craniotomy may be met in any of these phases.

No medications are usually administered during the mapping phase. The choice of technique may vary and must consider the team preference, location of the lesion, neurological status, body size, age, motivation, medical comorbidities, and patient's physical condition. To achieve smooth transitions and facilitate intra-operative mapping, anesthetics need to have a rapid onset, offset easy titratability, and minimal lingering effects. The most commonly used agents are Propofol, Fentanyl, Remifentanil, Dexmedetomidine (DEX), and Sevoflurane.

This technique has been adopted successfully with the availability of short-acting, titratable, and rapidly cleared anesthetics such as Propofol and Remifentanil. ${ }^{7}$ Aside from the easy titratability of Propofol and rapid recovery profile, it is also associated with a decreased cerebral metabolic rate for oxygen and intracranial pressure. Moreover, it has been shown to possess some neuroprotective, anticonvulsant, and antiemetic properties. In contrast, Remifentanil is an ultrashort acting opioid that also makes titration meet individual anesthetic requirements relatively more straightforward. A target-controlled infusion (TCI) function is present with both drugs, which allows for better drug titration and a predictable time for arousal after prolonged infusion.

Although both drugs have very desirable properties, they also have adverse effects, including dose-related respiratory depression, especially in patients with unsecured airways. ${ }^{8}$ It has been reported that respiratory complications occur in $18 \%$ of patients under propofol and Fentanyl/Remifentanil anesthesia. There is a significantly greater incidence of respiratory adverse events in patients under PropofolRemifentanil versus Dexmedetomidine anesthesia in another study. Also, oversedation may result in apnea, airway obstruction, hypoxemia, hypercapnia, and cerebral swelling. Therefore, constant monitoring of respiratory function clinically guided by the end-tidal $\mathrm{CO}_{2}$ is essential. On the other hand, undersedation may lead to agitation, restlessness, hypertension, and tachycardia. ${ }^{9,10}$ The use of sedation scales such as the Ramsay sedation score has helped ensure an appropriate depth of anesthesia throughout the procedure. ${ }^{11,12}$

The disadvantages of the procedure identified are as follows: (1) possibility of a prolonged surgical procedure with awkward body positioning; (2) need for patient cooperation to evaluate language, memory, and motor skills; (3) potential for significant patient discomfort, e.g., pain, nausea and recall; and (4) potential for emergent airway management should the patient become uncooperative or when persistent seizure activity occurs. Knowledge of these consequences is the first step in addressing them during the awake phase of the procedure.

Basic knowledge of neurologic testing and its consequences will dictate the success of an awake craniotomy because the sudden termination of the procedure may be influenced by factors brought about by neurostimulation during testing.

The commonly anticipated challenges during the awake phase may be surgical, medical, or anesthetic. The decision to change the surgical course and the anesthetic technique of an awake craniotomy procedure will address the issues without compromising the patient. The awake craniotomy technique may be classified as a failure if the awake neurologic testing cannot proceed safely because of unresolved challenges met during this phase of the surgery. This decision will have to be made upon by both the neurosurgical and anesthesia team.

\section{REPORT OF CHALLENGES IN THREE (3) AWAKE CRANIOTOMY CASES}

In this article, challenges in the performance of three (3) awake craniotomy cases are reported. The first case shows a surgical challenge due to an intra-operative finding. The second case focuses on neuroanesthesia challenges, particularly the airway compromise and neurologic deterio- 
ration during the procedure. The third case discusses the acute neuro-critical care challenge met by the patient who developed focal seizures during an awake neurologic testing. The cases presented correspond to significant difficulties for the neuroanesthesiologist due to the sudden shift in surgical and anesthesia plans with patient safety in consideration. Failure in the technique is defined as the inability to perform intra-operative neurologic mapping successfully during the procedure. The incidence of failed awake craniotomy in the PGH will be discussed. Factors leading to awake craniotomy failures will be identified, and how the situations were managed will be reviewed.

\section{Case 1: A Surgical Challenge: Failed Awake Craniotomy for Clipping of a Giant Left Middle Cerebral Artery Aneurysm}

A 45-year-old, right-handed, ASA 2 (American Society of Anesthesiologists), 70kg, GCS 15 (Glasgow Coma Scale) male presented with a brief generalized tonic-clonic seizure for the first time during a routine medical check-up. MRI scan of the brain demonstrated a mass compatible with a cavernoma at the left temporal lobe. A week later, he was then scheduled for an awake craniotomy to excise the cavernoma (Figure 1) under TIVA (total intravenous anaesthesia) asleep-awake-asleep technique.

The patient was premedicated with midazolam (0.05 $\mathrm{mg} / \mathrm{kg}$ IV) but remained awake and conversant before anesthesia. Basic ASA monitors, arterial lines, and condom urinary catheters were put in place. After a comfortable position was established, topical Lidocaine spray was initiated to anesthetize the airway adequately. The depth of sedation was kept within mild to deep stages depending on every stage of the procedure. The patient was then sedated with fentanyl (1 ug/kg) IV and propofol $(1-2 \mathrm{mg} / \mathrm{kg})$ IV, and LMA (laryngeal mask airway) was inserted with ease. Propofol $(50-75 \mathrm{mcg} / \mathrm{kg} / \mathrm{min})$ and Dexmedetomidine

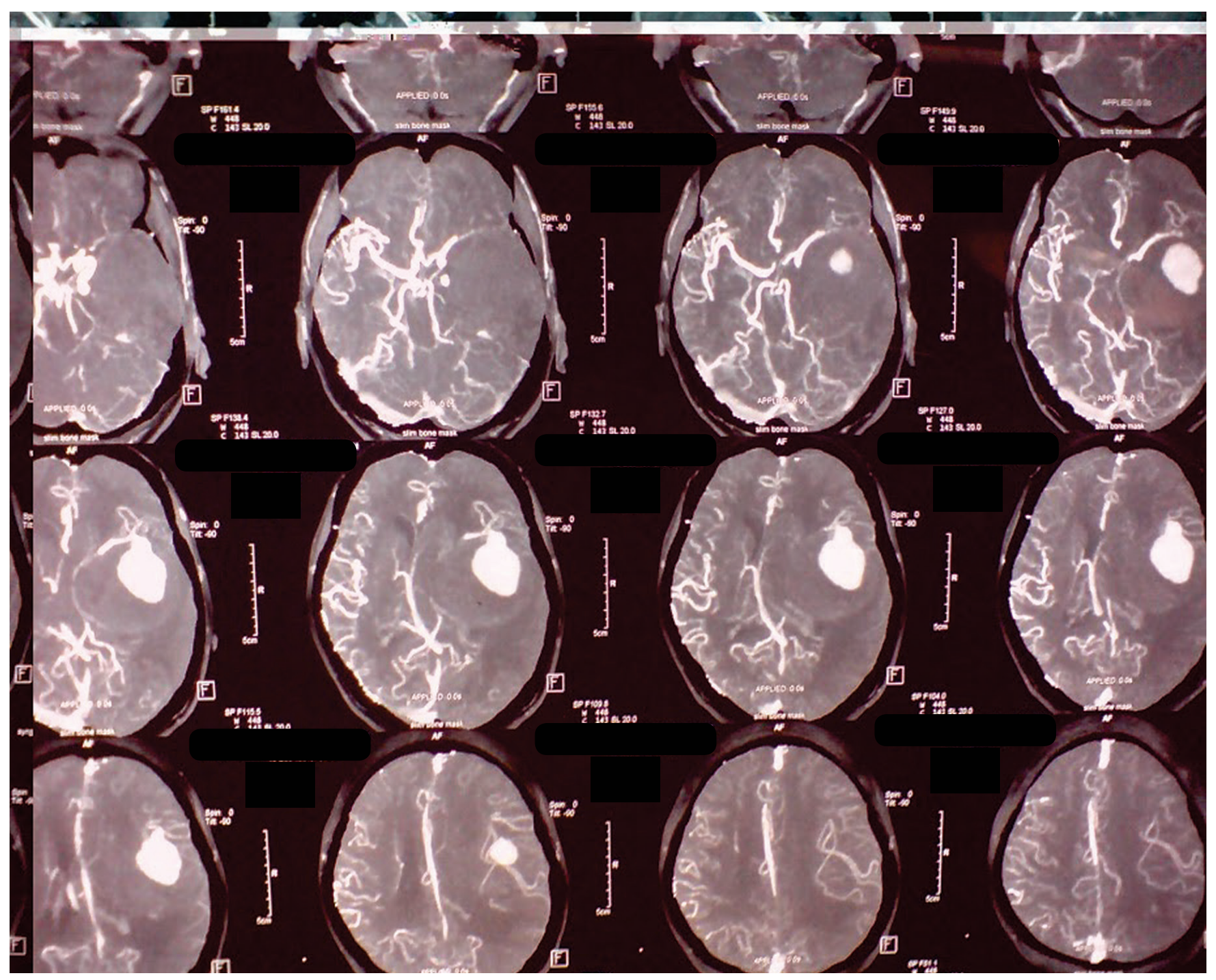

Figure 1. Brain MRI revealing a Left Temporal Cavernoma (5mm x 4mm). 
DEX $(0.2-0.3 \mathrm{mcg} / \mathrm{kg} / \mathrm{hr})$ infusions were started. $\mathrm{EtCO}_{2}$ (End-tidal carbon dioxide) maintained at $32-38 \mathrm{mmHg}$. Scalp nerve block was performed using $20 \mathrm{~mL}$ of Lidocaine $1 \%$ and Bupivacaine 0.25\% with Epinephrine 1:200,000 solution. The head was then secured using the Mayfield head frame, and after that, surgery commenced. Propofol and Dexmedetomidine (DEX) infusions were discontinued at 8 minutes and 40 minutes, respectively, before anticipating the patient waking up. The LMA was removed before the dura was opened.

The patient was then maintained on $\mathrm{O}_{2}$ support via nasal cannula at Fi02 of 0.3. The patient was breathing spontaneously, and the cortical surface was noted to be mildly bulging. A 30-degree head-up was done, and voluntary hyperventilation was encouraged to a targeted $\mathrm{EtCO}_{2}$ of 30-35 $\mathrm{mmHg}$. The procedure continued with the patient awake and oriented. Further exploration revealed a giant Left Middle Cerebral Artery (MCA) aneurysm (Figure 2) instead of a cavernoma. The neurosurgery team informed all the other services regarding their intra-operative finding. A temporary clip application was attempted on the aneurysm's proximal end while the patient was encouraged to speak. Slurring of speech was detected in less than one (1) minute after applying the temporary clip with rapid recovery after clip removal. The neurosurgical team decided to abandon the awake craniotomy technique with plans for further evaluation with a four-vessel cerebral angiography. Low dose infusions of Propofol $(50 \mathrm{mcg} / \mathrm{kg} / \mathrm{min})$ and Dexmedetomidine DEX $(0.2 \mathrm{mcg} / \mathrm{kg} / \mathrm{hr})$ were resumed until the end of the surgery with the patient lightly sedated, conversant, and breathing spontaneously with the nasal cannula at $\mathrm{O}_{2}$ at $4 \mathrm{~L} / \mathrm{min}$.
The patient was awake, oriented, and with no neurological deficits after the procedure was aborted. There was no seizure episode noted. Within that week, he was re-scheduled for an open craniotomy and clipping of the left middle cerebral artery aneurysm, which progressed successfully without any neurologic compromise postoperatively.

Ideally, the clipping of giant aneurysms is done under general anesthesia with the patient intubated because of the risk of cerebral compromise once the aneurysm is clipped. As a result, cerebral vasospasm may occur, which will compromise the blood supply in a large territory supplied by the particular major cerebral vasculature. When this happens, significant brain edema may occur, which will compromise the patient's sensorium and airway, which will be more difficult to handle during the awake craniotomy procedure. Early on, there was a change in anesthetic plans to avoid this from happening. The combination of Propofol and Dexmedetomidine infusion in low doses will decrease cerebral blood flow, $\mathrm{CMRO}_{2}$, and is able to provide adequate analgesia required for this operation.

Awake craniotomy for clipping of giant aneurysm is rare and has very few reported cases in the literature. Rapid assessment of neurologic changes was critical for this case, and the anesthetic technique used allowed the team to do this successfully. This report verified that appropriate patient selection and adequate patient preparation, knowledge of surgeon's skills and awareness of limitations, judicious use of anesthetics, and its adjuncts are necessary to have satisfactory results in this kind of operation. It is important to emphasize that a change in the surgical approach is warranted at any point of the procedure, especially when deemed unsafe to proceed with an awake surgical technique.

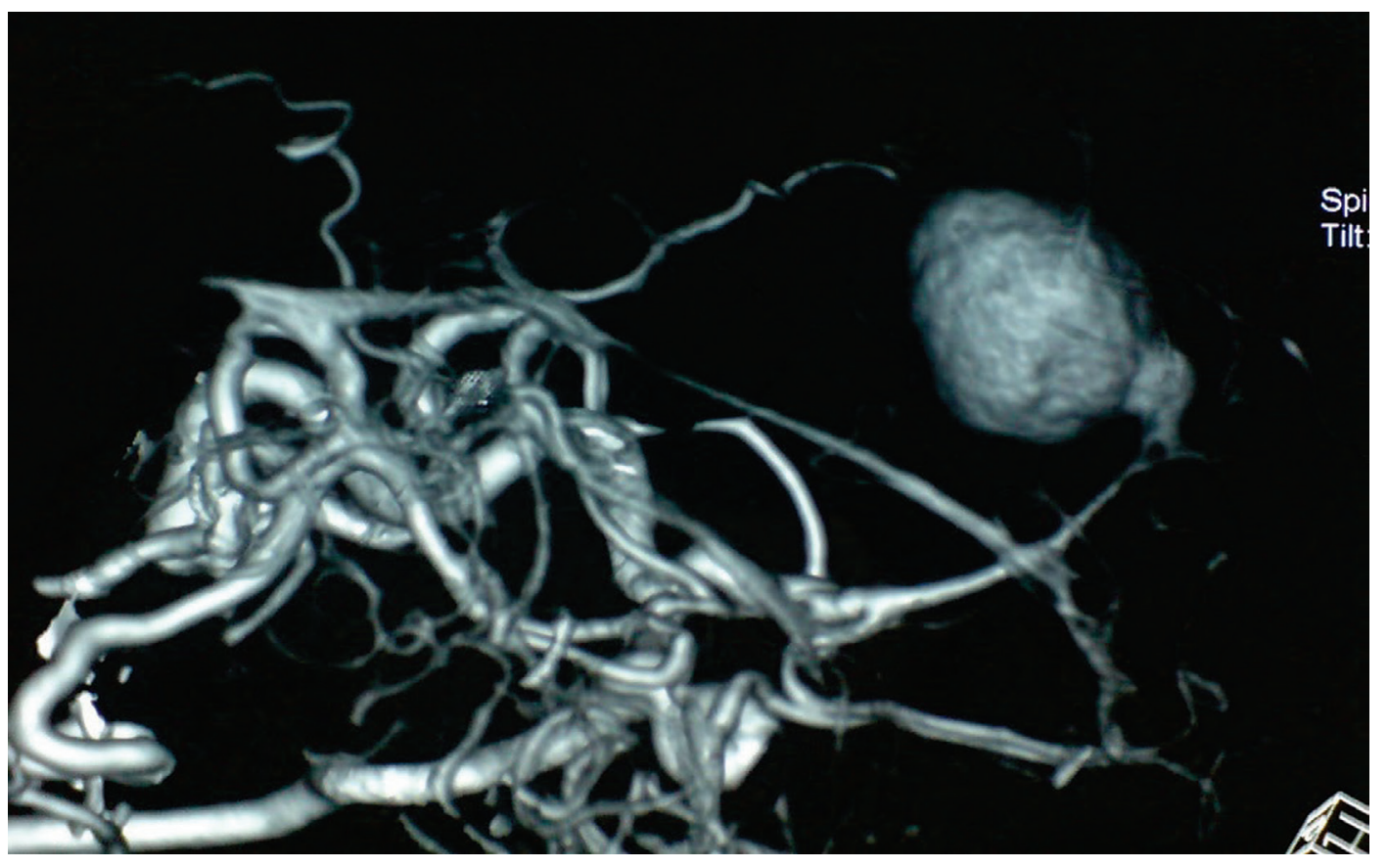

Figure 2. A 4-vessel angiography of the giant Left Middle Cerebral Artery Aneurysm (diameter 26mm). 
Case 2: A Neuroanesthesia Challenge: Failed Awake Craniotomy for Excision of a Left Temporal Arteriovenous Malformation

A 27-year-old 51kg, ASA 1, GCS 15 female and a physical therapist presented with a cerebrovascular tumor at the left temporoparietal area is for an awake craniotomy. She had a one-year history of a vague, throbbing left-sided headache and a brief episode of generalized tonic-clonic seizures controlled by the intake of Dilantin five (5) days before the scheduled procedure. There were no other neurologic deficits noted. Cranial MRI revealed a $6.1 \mathrm{~mm}$ x $5.4 \mathrm{~mm}$ x $4.8 \mathrm{~mm} \mathrm{AV}$ malformation at the left temporoparietal area. The feeding artery is the left middle cerebral artery, and the draining sinus is the left straight sinus. Laboratory findings were unremarkable. The anesthetic plan was TIVA asleepawake-asleep technique.

The surgery and anesthesia techniques were thoroughly explained and were accepted. The level of sedation and pain scores were determined at every stage of the procedure. The depth of sedation was classified as either mild, moderate, or deep depending on the requirements. Basic ASA monitors were placed, and a Hudson mask with $\mathrm{O}_{2}$ flow (fraction of inspired oxygen) at $5 \mathrm{~L} / \mathrm{min}\left(\mathrm{FiO}_{2} 0.4\right)$. She was requested to position herself at the operating table and asked to find the most comfortable position. This had a bearing on how she would wake up and cooperate during the awake neurologic testing. She was given premedication of midazolam 0.025 $\mathrm{mg} / \mathrm{kg}$ IV and fentanyl $0.5 \mathrm{ug} / \mathrm{kg}$ IV during this phase. Once the patient had positioned herself, TCI propofol at $2-3 \mathrm{ug} / \mathrm{mL}$ and Dexmedetomidine (DEX) at $0.3 \mathrm{ug} / \mathrm{kg} / \mathrm{hr}$ was started. Scalp block was then performed using $20 \mathrm{~mL}$ of Bupivacaine 0.25\% + Epinephrine 1:200,000 solution. Surgery commenced, and Propofol was discontinued 10 minutes before cortical mapping. Dexmedetomidine (DEX) was discontinued 30 minutes before the awake neurologic testing. The patient had spontaneous ventilation with an $\mathrm{EtCO}_{2}$ of 35 to 38 . The brain was noted to be adequately relaxed. We waited until the patient was fully awake and cooperative before we started with the neurologic testing. In her case, we tested for speech during the excision, requiring a fully awake patient, coherent and with all faculties intact. Cortical stimulation was done using 2 to $6 \mathrm{~mA}$. She was asked to recite the alphabet, date, time, location, and asked to talk about herself, her name, address, and job description. Her vital signs were stable at BP 120/70, HR 68, RR 12 with $\mathrm{O}_{2}$ saturation of $98 \%$ at $\mathrm{FiO}_{2}$ of 0.3 . She was able to perform this with ease; however, she suddenly became unresponsive during the AVM excision at the subcortical level. Her breathing pattern became deep and slow at a rate of 8 cycles $/ \mathrm{min}$. Pupils became anisocoric with left $>$ right. The $\mathrm{EtCO}_{2}$ suddenly increased to $46 \mathrm{mmHg}$, but $\mathrm{O}_{2}$ sat remained $97 \%$. The neurosurgeon was advised of this acute neurologic change and was asked to terminate the neurologic testing and the procedure while the patient was stabilized. This clinical presentation may suggest progressing cerebral edema during the excision of the AVM. The airway was then secured using an LMA supreme size 3 inserted in the right lateral decubitus position. A Propofol bolus of $1 \mathrm{mg} / \mathrm{kg} \mathrm{IV}$ was given to achieve this purpose. Giving a small bolus of an IV anesthetic is necessary to avoid airway stimulation that may lead to ascending or descending herniation. Assisted ventilation commenced after proper placement of the LMA verified by chest rise and decreased $\mathrm{EtCO}_{2}$ to 34 $\mathrm{mmHg}$. Once the ventilation had been secured, completion of the surgery commenced without the further benefit of the cortical neurologic testing. The vital signs of the patient were stable after that, and LMA was removed after the procedure. Blood loss was minimal. No blood transfusion was done. The patient emerged from anesthesia awake, coherent, oriented with slight slurring of speech. The patient stayed admitted for seven (7) days in the neurointensive care unit and recovered neurologically. MRI taken on postoperative day 2 showed complete excision of the AVM. Excision of an AVM results in blood termination from the arterial to the cerebral circulation's venous side. The blood supply territory of a particular cerebral vasculature will dictate the degree of cerebral edema, which may occur after the excision. The larger the blood supply territory, the larger is the area of a possible compromise to the brain tissue supplied by the anomalous arterial vessel. The postoperative course of the patient will depend largely on how these flow changes can impact the brain.

In this case, the awake neurologic testing was terminated because the patient can no longer actively participate due to the acute neurologic deterioration. Early recognition by the neuroanesthesiologist of the evolving airway issues and neurologic damage needs a quick intervention to avoid compromising the patient. Immediate communication with the neurosurgeon is vital in ensuring patient safety.

\section{Case 3: An Acute Neuro-Critical Care Challenge: Failed Awake Craniotomy for Tumor Resection with a Focal Seizure Episode during Awake Neuro- logic Testing}

A 41-year-old $63 \mathrm{~kg}$, ASA 2, GCS 15, right-handed male with a BMI of 24.9 presented with two (2) episodes of brief tonic-clonic seizures resolved spontaneously within a one (1) year period. His brain MRI revealed a left frontoparietal lobe mass measuring $5.2 \mathrm{~cm} \mathrm{x} 4.3 \mathrm{~cm} \mathrm{x} 8 \mathrm{~cm}$ with minimal peri-lesional edema (Figure 3). He was programmed for an awake craniotomy and tumor resection for low-grade glioma at the left frontoparietal lobe. His seizures were described as a brief loss of consciousness lasting for 2-3 minutes without a post-ictal period. His cranial nerves were intact, with no motor or sensory deficits. Since the consult, he had been taking Levetiracetam $500 \mathrm{mg}$ OD regularly. $\mathrm{He}$ had no previous surgical procedure and denied drug or alcohol abuse. Other preoperative laboratory results were normal. Airway assessment and the physical examination were unremarkable.

In the preoperative stage, the planned procedure was explained thoroughly, including the possible intra-operative 


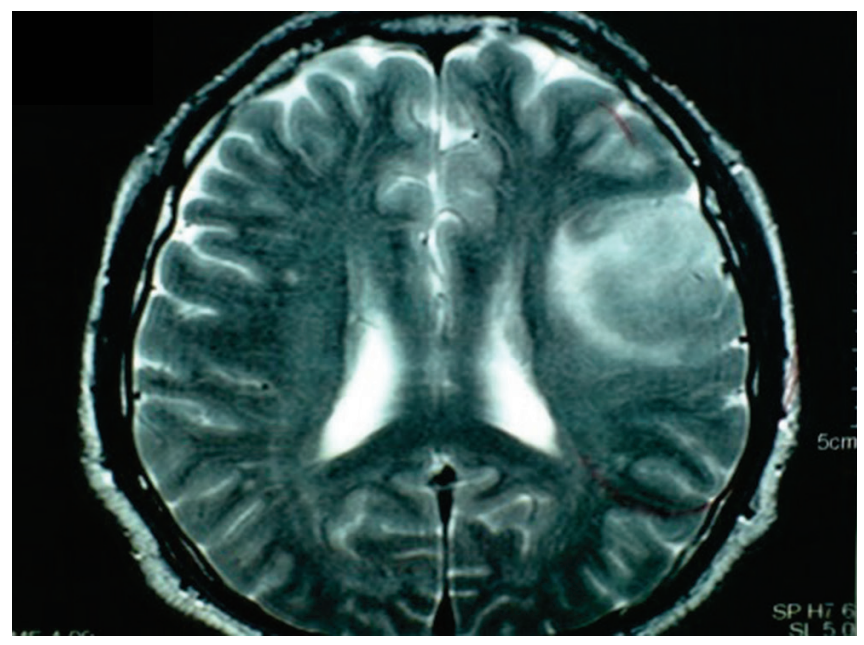

Figure 3. Brain MRI showing a Left Temporo-Parietal Tumor with minimal peri-lesional edema.

events. The patient was informed of the details of the anesthetic technique, the risks, and benefits. He also received a neurological and psychological evaluation. He was advised about his important role during the awake neurologic testing. Fortunately, the patient is very cooperative and collaborative and is aware that his active participation is vital for the operation's success. After providing all the necessary information and addressing all concerns, the patient gave his consent, and the surgical plan proceeded the next day. The patient was then brought to the operating room, awake, calm, and breathing spontaneously. Basic ASA monitors were placed, including a five lead electrocardiogram, pulse oximetry, temperature, and a radial arterial line in the lesion's contralateral side. His initial vital signs were: BP 120/70, HR 90bpm, RR 12cpm, $\mathrm{T}$ of $36.2 \mathrm{C}$, and $\mathrm{O}_{2}$ sat $97 \%$. Supplemental oxygen was provided via a $\mathrm{FiO}_{2}$ nasal cannula set at 0.3. The anesthetic plan was TIVA asleep-awake-awake technique using Propofol TCI and Remifentanil TCI.

A target-controlled infusion (TCI) of Propofol using Schnider's model was used at $1 \mathrm{ug} / \mathrm{mL}$. Remifentanil TCI was started using Minto's model at $0.4 \mathrm{ng} / \mathrm{mL}$. Schnider's Propofol model is a pharmacokinetic plasma model of TCI pumps, which requires the age, height, and the total body weight to be input for programming. The pump calculates the lean body mass (LBM) for the patient and adjusts the doses and infusion rates accordingly. This will result in lower Propofol infusion requirements, decreasing the total cost of the drug use.

On the other hand, Minto's model of Remifentanil is a pharmacokinetic model specific to Remifentanil. It is a useful predictor of its concentration in plasma and the effect site. This will then translate to faster and earlier patient recovery from the drug. The Ramsay sedation scale (Table 1) was used to know the depth of anesthesia provided to the patient. Ventilation and $\mathrm{CO}_{2}$ were monitored using a capnography line $\left(\mathrm{EtCO}_{2}\right)$ attached to the nasal cannula. The patient was then positioned right semi-laterally. Scalp block was performed using $40 \mathrm{~mL}$ of Bupivacaine $0.25 \%$ with 1:200,000 epinephrine. An anesthetic blockade of the pin site insertion was performed for cranial stabilization with a Mayfield holder. Drapes were placed and adjusted for easy accessibility of the airway and extremities during mapping.

Propofol and Remifentanil TCI values were adjusted to maintain a Ramsay sedation score of 2-3 (Table 1). Neuronavigation was initiated for delimitation of the intracranial lesion before the left pterional craniotomy. Upon skin incision, there were minimal changes in blood pressure and heart rate. Ramsay score was $5-6, \mathrm{O}_{2}$ saturation was $97-98 \%$ with an adequate respiratory pattern. TCI values for Propofol and Remifentanil were at $1 \mathrm{ug} / \mathrm{mL}$ and $0.4 \mathrm{ng} /$ $\mathrm{mL}$, respectively. Frequent monitoring of sedation depth was necessary during scalp and bone flap elevation to ensure the patient's comfort. During bone work, Remifentanil TCI was increased to $1.5 \mathrm{ng} / \mathrm{mL}$ at the effect site. Field block of the dura was then done with $1 \mathrm{~mL}$ of $2 \%$ Lidocaine. At this time, Propofol infusion was discontinued, and Remifentanil was decreased to $0.2 \mathrm{ng} / \mathrm{mL}$.

Once the dural opening was completed, and approximately 10 minutes after the IV anesthetics were stopped, the patient was awake and oriented. A Ramsay score of 2 was maintained so that the patient could cooperate and follow commands during the neuronavigation, functional testing, and tumor resection. Motor testing was done, and the patient was asked to move his fingers and extremities. During the neurologic testing, the cortical stimulation was gradually increased from $2 \mathrm{~mA}$ to $8 \mathrm{~mA}$ by increments of 2 $\mathrm{mA}$ until a response is noted. Either an involuntary or loss of movement of the particular extremity are observed. Upon cortical stimulation in the tumor site using $8 \mathrm{~mA}$, the patient suddenly went into focal seizures of the right upper extremity. The neurosurgeon was advised immediately about this, and

Table 1. Changes in Effect-Site Concentrations of Propofol and Remifentanil

\begin{tabular}{lcccc} 
& Propofol TCl $(\mu \mathrm{g} / \mathrm{mL})$ & Remifentanil $(\mathrm{ng} / \mathrm{mL})$ & ${ }^{*}$ RSS & ${ }^{*}$ SpO $_{2}$ \\
Application of Mayfield & 1 & 0.4 & 2 & 97 \\
Skin Incision & 1.5 & 0.4 & 4 & 98 \\
Cortical Mapping & 0 & 0.2 & 2 & 99 \\
Tumor Resection & 0 & 1.0 & 2 & 99 \\
After Tumor Resection & 1 & 0.4 & 6 & 99 \\
\hline
\end{tabular}

${ }^{*} \mathrm{RSS}=$ Ramsay Sedation Scale; $\mathrm{SpO}_{2}=$ oxygen saturation 
during this time, the patient was not following commands. Vital signs were stable and revealed a BP 130/70 HR 96/ min RR 24/min $\mathrm{EtCO}_{2}$ of $46 \mathrm{mmHg}$. Breathing was shallow and fast. The patient was given a bolus of Propofol at $1 \mathrm{mg} /$ $\mathrm{kg}$ IV, and the brain surface was flooded with ice-cold normal saline and covered with wet sterile gauze. Airway and ventilation were observed. The $\mathrm{O}_{2}$ saturation was still maintained between 97 to $99 \%$ at $\mathrm{FiO}_{2}$ of 0.4 .

The awake neurologic testing and the surgery was stopped while the patient was unstable. The seizure episode has resolved, and Propofol TCI was restarted at $1 \mathrm{ug} / \mathrm{mL}$. An LMA was then inserted in a right lateral decubitus position, and ventilation was assisted to a targeted $\mathrm{EtCO}_{2}$ of $32-35 \mathrm{mmHg}$. Remifentanil TCI was increased to $0.5 \mathrm{ng} /$ $\mathrm{mL}$. After discussing the risks and benefits of proceeding with the surgery, the team decided to proceed without the added advantage of an awake neurologic testing. After the tumor's gross total excision (Figure 4), all IV anesthetics were titrated to the desired Ramsay scale to close the craniotomy flap. After the surgery, the LMA was removed carefully and was shifted to $\mathrm{O}_{2}$ support with the nasal cannula at $\mathrm{FiO}_{2}$ of 0.3 . The patient was transferred to the neurosurgical intensive care unit fully awake, conversant, calm, and without pain. Postoperative Brain MRI confirmed the complete excision of the tumor. The rest of the peri-operative course was uneventful and he was discharged on the 6th post-op day.

The cortical surface is susceptible to current applied to it, especially in the temporal lobe. It may disrupt the synchronicity, flow, and movement of ions across the neuronal cells, resulting in a seizure episode. This is the reason why it was gradually adjusted by increments of $2 \mathrm{mAmps}$ to achieve the goal of identification of the cortical area responsible for a particular sensory or motor activity. The first response, when faced with this issue, is to inform the neurosurgeon about the incident. He will then flood the cortical area stimulated with cold saline solution to slow down the cortical

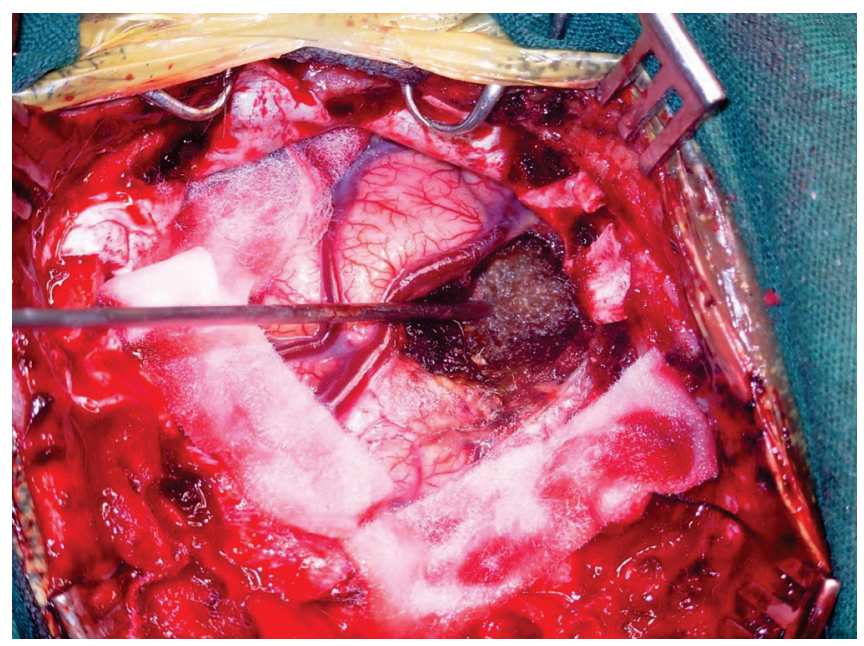

Figure 4. Post-excision of the tumor after a pre-terminated awake neurologic testing. stimulation's conduction on the brain surface. On the part of the anesthesiologist, he must give a fast-acting anti-epileptic drug to decrease the $\mathrm{CMRO}_{2}$ requirements of the brain and secure the airway with LMA to reduce the rising $\mathrm{CO}_{2}$ levels, which may lead to cerebral edema. In this case, the reason for proceeding with the surgery without the neurologic testing is, by weighing the risk vs. the benefit of continuing with the operation dictated that it is more beneficial to proceed to avoid the occurrence of further seizure episodes that may compromise the patient which may continuously occur after the surgery. It is therefore considered a failed awake craniotomy. The anesthesiologist must be ready for this acute clinical deterioration. He must be aware that this may happen any time during the neurostimulation and must know how to handle this issue so that a shift in anesthetic plans can be made at the right time for the patient's safety.

Although awake craniotomy procedures have been expanding its role in the neurosurgical field, there is still limited evidence on the optimal anesthetic management. ${ }^{13,14}$ Several protocols have been published; however, no single pharmacologic regimen has been recommended. ${ }^{15}$ It is essential to find a technique that provides not only adequate sedation, analgesia, respiratory and hemodynamic control, but also an awake and cooperative patient for neurological testing during the cortical mapping stage. ${ }^{16}$

Intra-operative complications are not uncommon; specific preventive measures and monitoring are mandatory for the safe performance of awake craniotomy. In addition to all routine monitoring, regular clinical assessment of the breathing pattern, depth, and rate supported by the endtidal $\mathrm{CO}_{2}$ measurements are mandatory for all patients. Optimizing the head position is vital for better cerebrospinal fluid drainage, accessibility of the airway in emergencies, and good communication with the patient during the mapping procedure. Preparation for the possible intubation intraoperatively must be anticipated. Equipment such as laryngeal mask airway (LMA), endotracheal tube, laryngoscopy, and medications should be prepared for conversion to GA. Fiberoptic bronchoscopy should also be readily accessible.

Additionally, intra-operative seizures are mostly focal seizures related to the cortical stimulation and usually resolves spontaneously after the cessation of stimulation. However, ice-cold saline applied by the surgeon to the brain surface and small doses of anticonvulsants may be required in some cases. All of these measures were observed in this patient.

In the PGH (2006-2019), there were 5/72 (7\%) awake craniotomy procedures that were aborted intra-operatively because of the following reasons: (a) positioning issues: too much flexion of the neck leading to airway compromise $-2 / 5$ (40\%); (b) continuous focal seizures - 1/5 (20\%); (c) hyper gag response - 1/5 (20\%); (d) overly anxious patient- 1/5 (20\%). No situation led to a significant patient compromise.

In a similar study, the failure rate of awake craniotomies is $6.4 \%$. The leading cause of failure was the lack of intraoperative communication with the patient $(4.2 \%)$ and intra- 
operative seizures (2.1\%). History of seizures $(p=0.03)$ and treatment with multiple anti-epileptic drugs $(\mathrm{p}=0.0012)$ was found to be related to failure due to intra-operative seizures. ${ }^{17}$ It has also been reported that failures in awake craniotomy were associated with a lower incidence of gross-total resection ( $83 \%$ vs. $54 \%, \mathrm{p}=0.008)$, there was a higher incidence of short-term speech deterioration postoperatively (6.1\% vs. $23.5 \%, \mathrm{p}=0.0017)$ as well as at three months postoperatively ( $2.3 \%$ vs. $145.4 \%, \mathrm{p}=0.0002)$, and the hospitalization period was longer $(4.9 \pm 6.2$ days vs. $8.0 \pm$ 10.1 days, $\mathrm{p}<0.001)$. Notably, more significant complications occurred in the failure group (14.8\%) than in the successful group (4\%) $(\mathrm{p}=0.037)$. In the awake craniotomy cases done in PGH, these were not observed. It has also been stated that the majority of awake craniotomy failures were preventable by adequate patient selection and avoiding the side effects of drugs administered during surgery. ${ }^{17,18}$

Awake craniotomy is a demanding procedure that requires the neurosurgeon's constant watchful eye and, most significantly, the anesthesiologist. It takes a lot of experience for an anesthesiologist to consider this a safe procedure because it takes a thorough understanding of the surgical technique to anticipate and appropriately manage its possible detrimental consequences. As of date, the awake craniotomy cases in the PGH seem to show no serious adverse events which compromised patients' safety. Proper patient preparation and planning for anticipated consequences of every stage of the procedure made the difference.

\section{CONCLUSION}

The challenges met in an awake craniotomy performance will vary depending on the neurosurgeon, neuroanesthesiologist, and the patient in acute clinical condition. These were described in full detail in every case discussed. We have learned several lessons along the way as we continue performing the PGH's awake craniotomy technique. The planning for the procedure is as vital as its progress. Here is the summary of essential points from the neurosurgeon, neuroanesthesiologist, and patient perspective, contributing to an awake craniotomy's success or failure. These are as follows: (1) A thorough preoperative evaluation is vital for this procedure to ease the patient's fears and anxiety. Good communication among the OR team members is essential before and during the awake craniotomy procedure; (2) Proper patient selection and appropriate consideration of his condition are vital; (3) In positioning for surgery, we should listen to our patient. He can give valuable input regarding his level of comfort in a particular position. The patient must be relaxed and at ease before the administration of the anesthetic so that when it is time to wake up, he knows precisely how his whole body is oriented; (4) A thorough understanding of the "surgical landscape" of the procedure will prepare the surgical and anesthesia teams in providing the optimal environment for the patient as he embarks on his journey of the procedure. The preparedness of a multidisciplinary team is essential to address successfully the issues that may arise intraoperatively. Good communication is critical; (5) The neurosurgeon must also be aware of his limitations for the safety of the patient in an awake craniotomy; (6) The choice of the anesthetic technique, as well as sufficient depth of anesthesia, must be achieved as required at every phase of the procedure has to be anticipated (e.g., during head-pin placement, positioning, opening and closing of the bone flap and meninges). Considering the patient's sensitivity to the anesthetic cannot be overemphasized; (7) Lifting of the craniotomy flap (periosteum and dura) is the most painful stimuli of the procedure. An adequate regional scalp block and dural field block are necessary; (8) The dura must be opened after the patient has woken up and the airway device removed to prevent the patient from coughing. This is a critical phase since wrong timing may lead to a devastating outcome (e.g., herniation); (9) Waking the patient up can be a pleasant experience for both the patient and the team. It depends mostly on patient preoperative preparation, rapport with the team, and the anesthetic technique's suitability; (10) Full consciousness must be reached before cortical mapping, especially during speech and language testing. For motor testing, the patient can be lightly sedated enough to follow commands; (11) There must be a smooth transition between anesthesia and consciousness; (12) Adequate ventilation and oxygenation must be achieved using various airway devices. (13) From a surgical point of view, the brain is grossly "more relaxed" with a total intravenous anesthesia (TIVA) technique; (14) Patient satisfaction and wellbeing seem to be reasonable compared to GA-inhalational technique; and (15) Patient immobility and comfort throughout surgery is essential in the success of the operation.

In the local setting, the following factors need to be considered before performing an awake craniotomy procedure. These are: (1) adequate equipment must be available, including cortical brain stimulator, ultrasound, infusion pumps, airway equipment, and devices. (2) A proper surgical indication is an essential requirement (3) Appropriate patient selection and psychological preparation are vital in this type of surgery. (4) Sound surgical and anesthetic plans. (5) Good communication between patients, the surgical and anesthesia teams. (6) Reasonable anticipation of complications and their management will define this procedure's outcome, and (7) A certain confidence level must be gained by the neurosurgeon and anesthesiologist in performing such a procedure to make a sound judgment be arrived at as a team when complications arise. This is not a time to experiment because the patient can easily decompensate. The anesthesiologist must be ready with a strategy to intubate the patient and convert to general anesthesia if the need arises. It is in the spirit of cooperation, consultation, and collaboration among colleagues (neurosurgeon, neuroanesthesiologist, neurologist, neurointensivist, neuroradiologist, a neurophysiologist, and nurses) 
to provide the ideal surgical conditions for the patient to ensure the success of the operation. Neuroanesthesia perhaps has represented a subspecialty of anesthesia where the anesthesiologist's expertise and competence can immensely influence the patient's outcome. As the awake craniotomy technique becomes well established, the anesthetic management will continue to differ in each institution. Complications, especially related to the airway and respiration, will occur in some patients; however, appropriate management can be readily accomplished by proper anticipation and continuous vigilance. Indeed, neurosurgery advances, neurointensive care, and neuroradiology have only been possible due to the concomitant advances in neuroanesthesia. The refinement and evolution of this surgical and anesthesia technique continue, and the availability of novel drugs and monitoring modalities represents both an opportunity and a challenge. They represent an opportunity because novel monitoring modalities provide exciting insights into the disease mechanisms and pathophysiology and allow a specific selection of appropriate therapies. They also represent a challenge because there is a danger that we may confuse the aim of improved clinical management with the technological means of achieving it.

\section{FUTURE DIRECTION AND RESEARCH OPPORTUNITIES}

Failed awake craniotomy was associated with a lower incidence of gross total resection and increased postoperative morbidity. The majority of the challenges met in an awake craniotomy procedure may be preventable by adequate patient selection, knowing when to shift to an open craniotomy and good communication between the neurosurgeon and anesthesiologist. It is also essential to explore the relationship between awake craniotomy failures on the chosen anesthesia techniques. In a small population trial, an asleep-awakeasleep technique and Monitored Anesthesia Care (MAC) techniques were feasible and safe, whereas data for the awakeawake-awake technique are sparse. A large randomized controlled trial (RCTs) are required to prove the superiority of one anesthetic regimen over the other. The potential for surgical and anesthetic resource conservation in performing an awake craniotomy procedure can be a profitable research endeavor, especially in low-income countries. Outcomebased researches comparing GA-Inhalational versus MAC sedation for an awake craniotomy procedure and the impact of specific anesthetic agents on brain tumor resection are needed, especially its applicability in our local setting. A systematic analysis of the conversion rate from an initial plan of an awake craniotomy to the conventional craniotomy procedure due to pre-specified outcomes which may include the following: incidence of airway compromise, hypoxia, intra-operative seizures, neurologic dysfunction, failure in awake neurologic testing and conversion to general anesthesia can be further reviewed. A patient satisfaction rate for this type of procedure is also essential to explore. Identifying the factors that may contribute to making the patient's experience pleasant will benefit the patient. Despite many studies comparing short-term outcomes with different anesthetics and analgesics, there is still a need for large randomized-controlled clinical trials that examine the most desired anesthesia technique for this procedure, analyze the conversion rate of anesthesia regimen, complications, and long-term outcomes including tumor recurrence, survival, and quality of life.

\section{Statement of Authorship}

Both authors participated in the data collection and analysis and approved the final version submitted.

\section{Author Disclosure}

Both authors declared no conflicts of interest.

\section{Funding Source}

None.

\section{REFERENCES}

1. Gerardo D. Legaspi, MD, FAFN. History of Awake Craniotomy in the Philippines. Lecture. January 2020. Department of Anesthesia, Philippine General Hospital.

2. Hansen E, Seemann M, Zech N, Doenitz C, Luerding R, Brawanski A. Awake craniotomies without any sedation: the awake-awake-awake technique. Acta Neurochir. 2013 Aug; 155(8):1417-24. doi: 10.1007/ s00701-013-1801-2.

3. Beez T, Boge K, Wager M, Whittle I, Fontaine D, Spena G, et al. Tolerance of awake surgery for glioma: a prospective European Low Grade Glioma Network multicenter study. Acta Neurochir. 2013 Jul; 155(7):1301-8. doi: 10.1007/s00701-013-1759-0.

4. Milian M, Tatagiba M, Feigl GC. Patient response to awake craniotomy - a summaryoverview. Acta Neurochir. 2014 Jun; 156(6):1063-70. doi: 10.1007/s00701-014-2038-4.

5. Sommer B, Grummich P, Coras R, Kasper BS, Blumcke I, Hamer HM, et al. Integration of functional neuronavigation and intraoperative MRI in surgery for drug-resistant extratemporal epilepsy close to eloquent brain areas. Neurosurg Focus. $2013 \mathrm{Apr}$; 34(4):E4. doi: 10.3171/2013.2.FOCUS12397.

6. Gabarros A, Young WL, McDermott MW, Lawton MT. Language and motor mapping during resection of brain arteriovenous malformations: indications, feasibility, and utility. Neurosurgery. 2011 Mar; 68(3):744-52. doi: 10.1227/NEU.0b013e318207a9a7.

7. Sokhal N, Rath GP, Chaturvedi A, Dash HH, Bithal PK, Chandra PS. Anaesthesia for awake craniotomy: a retrospective study of 54 cases. Indian J Anaesth. 2015 May; 59 (5):300-5. doi: 10.4103/ 0019-5049.156878.

8. Lobo FA, Wagemakers M, Absalom AR. Anaesthesia for awake craniotomy. Br J Anaesth, 2016 Jun; 116(6):740-4. doi: 10.1093/ $\mathrm{bja} / \mathrm{aew} 113$.

9. Goettel N, Bharadwaj S, Venkatraghavan L, Mehta J, Bernstein M, Manninen PH. Dexmedetomidine vs propofol-remifentanil conscious sedation for awake craniotomy: a prospective randomized controlled trial. Br J Anaesth, 2016 Jun; 116 (6):811-21. doi: 10.1093/bja/aew024.

10. Chui J. Anesthesia for awake craniotomy: an update. Rev Colomb Anestesiol. 2015 Feb; 43:22-8.

11. Prontera A, Baroni S, Marudi A, Valzania F, Feletti A, Benuzzi F, et al. Awake craniotomy anesthetic management using dexmedetomidine, propofol and remifentanil. Drug Des Devel Ther. 2017 Mar; 11:593-8. doi: 10.2147/DDDT.S124736. 
12. Bolzani ND, Junqueira DO, Ferrari PAPF, Ferrari AF, Gaia F, Tapajós CM, et al. Anesthesia for awake craniotomy: a case report. Braz J Anesthesiol, 2013 Nov-Dec; 63(6):500-3. doi: 10.1016/ j.bjane.2013.02.003.

13. Meng L, Han SJ, Rollins MD, Gelb AW, Chang EF. Awake brain tumor resection during pregnancy: decision making and technical nuances. J Clin Neurosci. 2016 Feb; 24:160-2. doi: 10.1016/ j.jocn.2015.08.021.

14. Hervey-Jumper SL, Berger MS. Maximizing safe resection of lowand high-grade glioma. J Neurooncol 2016 Nov; 130(2):269-82. doi: 10.1007/s11060-016-2110-4.

15. Flexman AM, Meng L, Gelb AW. Outcomes in neuroanesthesia: what matters most? Can J Anaesth. 2016 Feb; 63(2):205-11. doi: 10.1007/s12630-015-0522-9.
16. Wigmore TJ, Mohammed K, Jhanji S. Long-term survival for patients undergoing volatile vs IV anesthesia for cancer surgery: a retrospective analysis. Anesthesiology. 2016 Jan; 124(1):69-79. doi: 10.1097/ALN.0000000000000936.

17. Nossek E, Matot I, Shahar T, Barzilai O, Rapoport Y, Gonen T, et al. Intraoperative seizures during awake craniotomy: incidence and consequences: analysis of 477 patients. Neurosurgery. 2013 Jul; 73(1):135-40. doi: 10.1227/01.neu.0000429847.91707.97.

18. Nossek E, Matot I, Shahar T, Barzilai O, Rapoport Y, Gonen T, et al. Failed awake craniotomy: a retrospective analysis in 424 patients undergoing craniotomy for brain tumor. J Neurosurg. 2013 Feb; 118(2):243-9. doi: 10.3171/2012.10.JNS12511.

Have you read the current trends in Medical and Health Research in the Philippines?

\title{
Acta Medica Philippina The National Health Science Journal
}

\author{
Access Online: www.actamedicaphilippina.upm.edu.ph
}

